\section{Understanding Pharmacology for Pharmacy Technicians (with workbook)}

Stuhan MA, editor. American Society of Health-System Pharmacists, Bethesda, Maryland, 2013. Main volume: softcover, 785 pages. ISBN 978-1-58528-2296. US\$67 (US\$60 for ASHP members). Workbook: softcover, 200 pages. ISBN 978-1-85828-3835. US \$29 (US\$23 for ASHP members). Package (main volume + workbook): ISBN 978-1-58528-3866. US\$86 (US\$75 for ASHP members).

Mary Ann Stuhan has compiled this set of pharmacology learning tools to help pharmacy technicians acquire knowledge to meet the accreditation standards of the American Society of Health-System Pharmacists and to aid in preparation for the US Pharmacy Technician Certification Board examination. Contributors and reviewers include clinical pharmacotherapy experts, pharmacy professors, pharmacy managers, and instructors and directors of pharmacy technician and technology programs.

Appropriately, the textbook opens with a chapter examining the practical and theoretical benefits of pharmacy technicians having an understanding of pharmacology. The remainder of the textbook contains 34 chapters organized by body systems. In addition to explaining disease processes, goals of therapy, and the pharmacology of medications used to treat various health conditions, the text expands on terms and definitions, includes tables outlining pharmacotherapy, and offers review questions and references at the end of each chapter. If an introduction to a particular body system is required to understand the pharmacology concepts, labelled colour pictures are provided to clarify the relevant anatomy and physiology. The text also provides clinical vignettes to help technicians understand the patient's perspective. Safety alerts and practice points highlight clinical, administrative, and product selection pearls. The layout makes the textbook easy to read. The content is indexed, and the main text links well to the accompanying workbook. Instructor resources (PowerPoint slides) are also available; however, they offer none of the practical aspects or practice points from the textbook and workbook, and rarely include helpful images to engage the viewer.

Despite the visually pleasing layout of both the textbook and the workbook, references to the policies of US drug regulatory authorities, as well as US brand names and manufacturers, limit the utility of this package for Canadian pharmacy technicians. In addition, many chapters provide anatomic, physiologic, or pharmacokinetic complexity that may exceed the needs or interests of technicians. For example, the description of pharmacokinetic concepts includes complex equations requiring scientific calculators and an advanced proficiency in mathematics. Each chapter contains multiple cases and associated questions; however, the layout makes it difficult to link the questions to particular cases. Appropriate auxiliary labelling and suggestions for handling medications would increase the practicality and applicability for technicians in the workplace.

This text and its associated workbook could serve as a resource for pharmacy technicians challenging licensing or qualifying examinations. It might also be useful for undergraduate students interested in exploring a career in pharmacy or as an addition to a pharmacy technician library, for reference or teaching purposes. Overall, this set of learning tools is best utilized together, as the workbook greatly increases relevance and practicality for a working pharmacy technician.

\section{Susan Balagus}

Practice Development Pharmacy Technician

Amy J Marriott, BSc, BScPharm, ACPR, PharmD

Practice Development Pharmacist

Winnipeg Regional Health Authority Pharmacy Program

Winnipeg, Manitoba

Competing interests: None declared. 